\title{
POLYANALYTIC FUNCTIONS WITH EQUAL MODULUS
}

P. KRAJKIEWICZ AND W. BOSCH

In [1], M. B. Balk obtains a representation for polyanalytic functions which have constant modulus on some region. Balk's result suggests the more difficult problem of obtaining necessary and sufficient conditions that two polyanalytic functions have equal modulus on some region. This problem is answered in Theorem 1 and as an immediate corollary to Theorem 1, we obtain the result of Balk cited in [1]. In Theorem 2 and subsequent corollaries, the question of establishing criteria that two polyanalytic functions have equal amplitudes or equal real parts or equal imaginary parts is considered.

In what follows, $G$ will denote a region of the finite complex plane $\Gamma$ and $G^{*}$ will denote the region $G^{*}=\{z \in \Gamma \mid \bar{z} \in G\}$.

Definition. A function $f: G \rightarrow \Gamma$ is said to be polyanalytic on $G$ or $n$-analytic on $G$ if and only if there exist $n \geqq 1$ functions $f_{0}, f_{1}, \cdots$, $f_{n-1}$ which are analytic on $G$ such that $f_{n-1}$ is not identically zero on $G$ whenever $n \geqq 2$ and such that $f(z)=\sum \bar{z}^{k} f_{k}(z)$, for all $z \in G$, where $k=0,1, \cdots, n-1$ and where $\bar{z}$ denotes the complex conjugate of $z$.

We now need the following preparatory lemma:

Lemma. Let $P$ and $Q$ be two nonidentically zero relatively prime polynomials in two variables such that $|P(z, \bar{z})|=|Q(z, \bar{z})|$, for all $z \in G$. Then there exists a complex constant $\lambda$ with $|\lambda|=1$ such that

$$
P(z, \bar{z})=\lambda \overline{Q(z, \bar{z})},
$$

for all $z \in G$.

Proof. If we let

$$
P_{1}(z, \bar{z})=\overline{P(z, \bar{z})} \text { and } Q_{1}(z, \bar{z})=\overline{Q(z, \bar{z})}
$$

for all $z \in \Gamma$, we see that $P_{1}(z, \bar{z})$ and $Q_{1}(z, \bar{z})$ are polynomials in $z$ and $\bar{z}$ and that

$$
P(z, \bar{z}) P_{1}(z, \bar{z})=Q(z, \bar{z}) Q_{1}(z, \bar{z}),
$$

for all $z \in G$. We now define the function $F$ by the condition that

$$
F(z, w)=P(z, w) P_{1}(z, w)-Q(z, w) Q_{1}(z, w),
$$

for all $z, w \in \Gamma$. Now $F(z, w)$ is a polynomial in $z$ and $w$ and $F(z, w)=0$ for all $z, w \in G$ which are on the manifold determined by the equation

Received by the editors March 4, 1969. 
$w=\bar{z}$. Hence it follows that $F(z, w)=0$ for all $z, w \in \Gamma[6$, p. 41]. Thus we see that

$$
P(z, w) P_{1}(z, w)=Q(z, w) Q_{1}(z, w),
$$

for all $z, w \in \Gamma$. Now $P(z, w)$ and $Q(z, w)$ are relatively prime and $P(z, w)$ divides $Q(z, w) Q_{1}(z, w)$. Hence there is a polynomial $R(z, w)$ such that $R(z, w) P(z, w)=Q_{1}(z, w)$, for all $z, w \in \Gamma$. Now $P_{1}(z, w)$ and $Q_{1}(z, w)$ are also seen to be relatively prime. Hence there is a polynomial $R_{1}(z, w)$ such that $R_{1}(z, w) Q_{1}(z, w)=P(z, w)$, for all $z, w \in \Gamma$. It then follows that $R(z, w) R_{1}(z, w)=1$, for all $z, w \in \Gamma$. Hence there is some constant $\lambda$ such that $R_{1}(z, w)=\lambda$ for all $z, w \in \Gamma$. Thus we see that $P(z, w)=\lambda Q_{1}(z, w)$, for all $z, w \in \Gamma$. Hence

$$
P(z, \bar{z})=\lambda \overline{Q(z, \bar{z})},
$$

for all $z \in G$. Clearly $|\lambda|=1$. This establishes the lemma.

In the sequel, we shall assume that $f, g: G \rightarrow \Gamma$ are two functions which are $n$-analytic and $m$-analytic, respectively, on $G$. Also if $P($,$) is a polynomial in two variables, let \partial P$ and $\delta P$ denote the degrees of $P$ in the first and second place variables, respectively.

We are now ready to state and prove Theorem 1:

Theorem 1. A necessary and sufficient condition that $|f(z)|=|g(z)|$, for all $z \in G$ is that there exist a nonidentically zero polynomial $P(z, \bar{z})$ in $z$ and $\bar{z}$ such that

$$
P(z, \bar{z}) f(z)=\overline{P(z, \bar{z})} g(z),
$$

for all $z \in G$. In the above condition we can also require that $\partial P \leqq(n-1)$ and $\delta P \leqq(m-1)$.

Proof. First it is clear that the condition is sufficient. It remains only to show that the condition is necessary. We need only consider the case when $f$ and $g$ do not vanish identically on $G$. In order to abridge notation somewhat we shall assume that $k=0,1, \cdots, n-1$ and $j=0,1, \cdots, m-1$. Now there exist functions $f_{k}(z)$ and $g_{j}(z)$ which are analytic on $G$ such that $f_{n-1}$ and $g_{m-1}$ do not vanish identically on $G$ and such that

$$
\begin{aligned}
& f(z)=\sum \bar{z}^{k} f_{k}(z), \\
& g(z)=\sum \bar{z}^{j} g_{j}(z),
\end{aligned}
$$

for all $z \in G$. We then have that

$$
\sum \bar{z}^{k} f_{k}(z) \sum z^{k} \bar{f}_{k}(\bar{z})=\sum \bar{z}^{j} g_{j}(z) \sum z^{j} \bar{g}_{j}(\bar{z})
$$


for all $z \in G$. In the above equation it is understood that

$$
\bar{f}_{k}(\bar{z})=\overline{f_{k}(z)} \text { and } \bar{g}_{j}(\bar{z})=\overline{g_{j}(z)}
$$

for all $z \in G$. We now introduce the auxiliary function $F$ defined by the condition that

$$
F(z, w)=\sum w^{k} f_{k}(z) \sum z^{k} \bar{f}_{k}(w)-\sum w^{i} g_{j}(z) \sum z^{j} \bar{g}_{j}(w),
$$

for all $(z, w) \in G \times G^{*}$. We see that $F(z, w)$ is analytic in $z$ and $w$ for all $(z, w) \in G \times G^{*}$. We also note that $F(z, w)=0$ for all $(z, w) \in G \times G^{*}$ such that $z$ and $w$ are on the manifold determined by the equation $w=\bar{z}$. Hence it follows that $F(z, w)=0$ for all $(z, w) \in G \times G^{*}[6$, p. 41]. Thus we have that

$$
\sum w^{k} f_{k}(z) \sum z^{k} \bar{f}_{k}(w)=\sum w^{j} g_{j}(z) \sum z^{j} \bar{g}_{j}(w),
$$

for all $(z, w) \in G \times G^{*}$. Since $f_{n-1}$ and $g_{m-1}$ do not vanish identically on $G$, we can find some $\left(z_{0}, w_{0}\right) \in G \times G^{*}$ and some $r>0$ such that $N_{1} \times N_{2}=N\left(z_{0}, r\right) \times N\left(w_{0}, r\right) \subseteq G \times G^{*}$ and such that each of the four sums in the previous equation are not zero for all $(z, w) \in N_{1} \times N_{2}$. We now introduce the auxiliary function $h$ defined by the condition that

$$
h(z, w)=\frac{\sum w^{k} f_{k}(z)}{\sum w^{j} g_{j}(z)}=\frac{\sum z^{j} \bar{g}_{j}(w)}{\sum z^{k} \bar{f}_{k}(w)},
$$

for all $(z, w) \in N_{1} \times N_{2}$. The function $h$ is clearly analytic and never zero on $N_{1} \times N_{2}$. Now for each fixed $z \in N_{1}$ we see that $h(z, w)$ is a rational function of $w$ and for each fixed $w \in N_{2}$ we see that $h(z, w)$ is a rational function of $z$. It therefore follows that $h(z, w)$ is a rational function of $z$ and $w$ [5, p. 35]. As indicated by Osgood in [5], this result follows from the last part of Hurwitz's proof $[3$, p. 151] of a theorem first stated by Weierstrass [7]. Hence there exist two nonidentically zero relatively prime polynomials $R(z, w)$ and $S(z, w)$ such that $S(z, w) h(z, w)=R(z, w)$, for all $(z, w) \in N_{1} \times N_{2}$. Hence we have that

$$
\begin{aligned}
& S(z, w) \sum w^{k} f_{k}(z)=R(z, w) \sum w^{j} g_{j}(z), \\
& S(z, w) \sum z^{j} \bar{g}_{j}(w)=R(z, w) \sum z^{k} \bar{f}_{k}(w),
\end{aligned}
$$

for all $(z, w) \in N_{1} \times N_{2}$. Now for all $z \in N_{1}$, with at most a finite number of exceptions, the expressions $R(z, w)$ and $S(z, w)$ considered as polynomials in $w$ are relatively prime $[2$, p. 210]. Hence from the above equations we see that $\delta R \leqq(n-1)$ and $\delta S \leqq(m-1)$. Similarly, $\partial R \leqq(m-1)$ and $\partial S \leqq(n-1)$. Observe also that the latter part of 
Hurwitz's proof of Weierstrass's theorem [3, p. 151], yields the same estimates as above on the degrees of $R(z, w)$ and $S(z, w)$ with respect to $z$ and $w$. From the previous equation we note also that $S(z, \bar{z}) f(z)$ $=R(z, \bar{z}) g(z)$, for all $z \in G$. Hence we see that $R(z, \bar{z})$ and $S(z, \bar{z})$ are two nonidentically zero relatively prime polynomials in $z$ and $\bar{z}$ which have equal modulus on $G$. Hence there is some constant $\lambda$ with $|\lambda|=1$ such that

$$
S(z, \bar{z})=\lambda \overline{R(z, \bar{z})},
$$

for all $z \in \Gamma$. Now $\lambda=e^{-2 i \theta}$ for some real number $\theta$. Let $P(z, \bar{z})=e^{i \theta} S(z, \bar{z})$ for all $z \in \Gamma$. Then $P(z, \bar{z})$ is a nonidentically zero polynomial in $z$ and $\bar{z}$ such that

$$
P(z, \bar{z}) f(z)=\overline{P(z, \bar{z})} g(z),
$$

for all $z \in G$. Moreover, $\partial P \leqq(n-1)$ and $\delta P \leqq(m-1)$. This completes the proof of the theorem.

From Theorem 1, we immediately obtain the following corollary:

Corollary 1. A polyanalytic function $f$ on $G$ has constant absolute value on $G$ iff there exists some constant $\lambda$ and some polynomial $P(z)$ in $z$ which is never zero in $G$ such that

$$
f(z)=\lambda \overline{P(z)} / P(z),
$$

for all $z \in G$.

We also have the following companion theorem to Theorem 1:

THEOREM 2. Assume that the polyanalytic functions $f$ and $g$ do not vanish identically on $G$. Then a necessary and sufficient condition that

$$
\overline{f(z)} g(z)=f(z) \overline{g(z)}
$$

for all $z \in G$ is that there exist two nonidentically zero real valued polynomials $A(z, \bar{z})$ and $B(z, \bar{z})$ in $z$ and $\bar{z}$ such that $A(z, \bar{z}) f(z)=B(z, \bar{z}) g(z)$, for all $z \in G$. In the above condition we can also require that $\partial A=\delta A$ $\leqq(m-1)$ and $\partial B=\delta B \leqq(n-1)$.

We omit the proof of Theorem 2 since it is similar to the proof of Theorem 1 in all essential details.

Note that Theorem 2 can be interpreted as giving a criterion for determining whether or not $f(z)$ and $g(z)$ have equal amplitudes modulo $\pi$ at the points $z$ of $G$ where neither function vanishes.

As an application of Theorem 2, we offer the following corollaries: 
COROLlaRy 1. Suppose that the polyanalytic functions $f$ and $g$ never vanish on $G$. Then

$$
\operatorname{amp} f(z)=\operatorname{amp} g(z)(\operatorname{modulo} 2 \pi),
$$

for all $z \in G$, if and only if the following condition holds: there exist two nonidentically zero real valued polynomials $A(z, \bar{z})$ and $B(z, \bar{z})$ in $z$ and $\bar{z}$ with $A B$ positive for at least one point of $G$ such that

$$
A(z, \bar{z}) f(z)=B(z, \bar{z}) g(z),
$$

for all $z \in G$. In the above condition we can require that $\partial A=\delta A \leqq(m-1)$ and $\partial B=\delta B \leqq(n-1)$.

Proof. From Theorem 2, it is clear that the condition is necessary. Now assume the condition. From Theorem 2, we see that amp $f(z)$ $=\operatorname{amp} g(z)(\operatorname{modulo} \pi)$ for all $z \in G$. Let $H$ denote the set of all points $z \in G$ for which amp $f(z)=\operatorname{amp} g(z)$ (modulo $2 \pi$ ) and let $K=G-H$. For $z_{0} \in G$, let $r>0$ be such that $N=N\left(z_{0}, r\right) \subseteq G$. Now there exist continuous functions $a_{1}$ and $a_{2}$ defined on $N$ such that $a_{1}(z)=\operatorname{amp} f(z)$ (modulo $2 \pi$ ) and $a_{2}(z)=\operatorname{amp} g(z)$ (modulo $\left.2 \pi\right)$ for all $z \in N$ [4, p. 328]. Since $\left(a_{1}-a_{2}\right) / \pi$ is a continuous integral valued function defined on $N$, this function reduces to some integral constant $k_{0}$ on $N$. If $z_{0} \in H$, then $k_{0}$ is even and hence $N \subseteq H$. However, if $z_{0} \in K$, then $k_{0}$ is odd and therefore $N \subseteq K$. Thus we see that $H$ and $K$ are both open sets. Since $G$ is connected and $H \neq \varnothing$, it follows that $G=H$. Thus the condition is sufficient. This completes the proof.

Corollary 2. Let $f$ and $g$ be polyanalytic on $G$. Then $f$ and $g$ have the same real part on $G$ if and only if there exists a real valued polynomial $A(z, \bar{z})$ in $z$ and $\bar{z}$ such that

$$
f(z)=g(z)+i A(z, \bar{z}),
$$

for all $z \in G$. Also $f$ and $g$ have the same imaginary part on $G$ if and only if there exists a real valued polynomial $A(z, \bar{z})$ in $z$ and $\bar{z}$ such that

$$
f(z)=g(z)+A(z, \bar{z}),
$$

for all $z \in G$.

\section{REFERENCES}

1. M. B. Balk, Polyanalytic functions of constant modulus, Litovsk. Mat. Sb. 6 (1966), 31-36.

2. M. Bôcher, Introduction to higher algebra, Macmillan, New York, 1922.

3. A. Hurwitz, Beweis des Satzes, dass eine einwertige Funktion beliebig vieler Variablen, welche überall als Quotient zweier Potenzreihen dargestellt werden kann, eine 
rationale Funktion ihrer Argumente ist, J. Reine Angew. Math. 95 (1883), 201-206 (Werke, Bd. I, 148-152).

4. C. Kuratowski, Théorèms sur l'homotopie des fonctions continues de variable complexe et leurs rapports a' la Théorie des fonctions analytiques, Fund. Math. 33 (1945), 316-367.

5. W. Osgood, Topics in the theory of functions of several complex variables, Dover, New York, 1966.

6. V. S. Vladimirov, Methods of the theory of functions of many complex variables, M.I.T. Press, Cambridge, Mass., 1966.

7. K. Weierstrass, Untersuchungen ïber die $2 r$-fach periodischen Funktionen mit $r$ Veränderlichen, Crelles Journal 89 (1880), 1-8 (Werke, Bd. II, 125-134).

UNIVERSITY OF NEBRASKA 